\title{
Analysis on static and dynamic performances of ultra-thin multi-lobe gas lubrication bearings
}

\author{
WU Datao 1, a, YANG Lihua 2,b \\ ${ }^{1,2}$ State Key Laboratory for Strength and Vibration of Mechanical Structures, Xi'an Jiaotong \\ University,Xi'an, Shaanxi 710049,China \\ 1,2Shaanxi Key Laboratory of Environment and Control for Flight Vehicle, Xi'an, Shaanxi \\ 710049 ,China \\ 1,2 School of Aerospace Engineering, Xi'an Jiaotong University, Xi'an,P.R. China \\ a1622617283@qq.com, byanglihua_2@126.com
}

\begin{abstract}
Keywords: rarefaction effect; modified Reynolds equation; PDE; static and dynamic performances Abstract. The coupling effects of gas-rarefaction are taken into consideration to investigate the static and dynamic performance of the three lobe gas bearing with ultra-thin gas lubrication. The influence of gas-rarefaction on static and dynamic performances of three-lobe bearing with different models are studied. The Reynolds equation is modified by the first order slip model,second order slip model and Boltzmann model, and corresponding journal perturbation equation are solved by MATLAB Partial Differential Equation(PDE) Toolbox. Effect of eccentricity, bearing number, length to diameter ratio and disturbance frequency on the flow characteristics, including gas film pressure distribution, load carrying capacity and eight linear dynamic coefficients, are discussed numerically. The results show that gas-rarefaction are of great significance in not only pressure distribution and load capacity but also bearing dynamic performance. The second-order and Boltzmann model are more consistent and is smaller than the result of first-order model.
\end{abstract}

\section{Introduction}

Hydrodynamic multi-lobe slider bearings are widely used in rotating machine systems such as high speed centrifugal air separator and gyroscope in the field of astronautic and aviation owing to its superior properties in oil whirl and oil whip than circular journal bearing, which has the features of high precision, low frictional power loss, high rotate speed and suit for large temperature range.The generalized Reynolds equation was derived based on the hypothesis of continuous flow might be not the case when the gas film thickness is close to the average molecular free path.The gas rarefaction effect must be taken into consideration[1-2], while studying the performance of gas slider bearing. The rarefied content of gas can be indicated by the Kundsen number, $K_{n}$, defined as the ratio of the average free path of gas molecule to the film thickness.

Burgdorger[3] first introduced first-order velocity slip boundary condition into Reynolds equation. Based on Burgdorger's modified Reynolds equation, the effect of temperature on the stiffness and damp coefficient of micro-gas journal bearing were discussed by Lee[4]. Hsia and Domoto[5]presented a second-order velocity slip modified Reynolds equation to obtain the performance of ultra-thin gas bearing.Mitsuya[6] proposed a modified Reynolds equation with 1.5-order boundary condition to investigate the gas lubricated bearing. The above three corrected models are precise only for small Kundsen number.Zhang Wenming et al [7-8] derived a numerical model with rarefaction and surface roughness effect, the result showed that the slip boundary condition can improve the stability of MEMS bearing-rotor system.Applying the linearized Boltzman equation, a lubrication equation can be obtained by Gans [4] first based on the BGK model[5], which is identical with the Reynolds equation corrected by the first-order model.The results show that this equation can be applied in wider range of Kundsen number.Based on a linearized Boltzman equation resemble Gans's ,Fukui and Kaneko[9-10] developed an improved Reynold's equation and applied the local interpolation method and coefficient database to accelerate its solution process. The results show that the load carrying capacities was overrated by applying first-order model, while underrated it 
by applying second-order model. Based on FK model, Zhang Xiaoqing et al.[11-12]studied the nonlinear dynamics characteristics of micro-gas spiral groove thrust bearing-rotor system. The results showed that the stability of bearing-rotor system was enhanced by considering the gas rarefaction effect. The most studies consider rarefaction effect by utilizing minimum film thickness in iterative calculation. However, the film thickness of the bearing is not a constant. A further research about actual gas film thickness on the performance computing of ultra-thin gas bearing is motivated.

In this paper, the different modified Reynolds equations with gas rarefaction is developed by incorporating with first-order, second-order and Boltzmann slip model. The influences of bearing number, eccentricity, length to diameter ratio and disturbance frequency on the static and dynamic characteristics of three lobe slider bearing are discussed in detail.Moreover, the results of first-order slip, second-order slip and Boltzmann modified model is compared.

\section{Mathematical model}

bearing structure.In this paper,three lobe bearing was taken as research object,and can be seen Fig.1

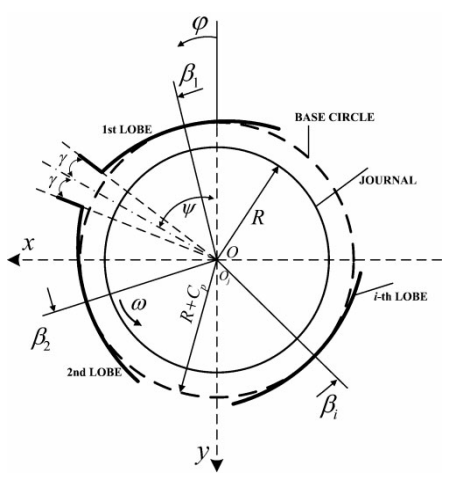

Fig.1 bearing structure

static performance.Under the condition of normal temperature,the dimensionless form of unsteady compressible fluid modified Reynolds Equation can be presented as[13]

$$
\frac{\partial}{\partial \varphi}\left(P H^{3} Q \frac{\partial P}{\partial \varphi}\right)+\frac{\partial}{\partial \lambda}\left(P H^{3} Q \frac{\partial P}{\partial \lambda}\right)=\Lambda \frac{\partial}{\partial \varphi}(P H)+2 \Lambda \frac{\partial}{\partial T}(P H)
$$

where $H=h / C_{b}\left(\mathrm{C}_{\mathrm{b}}\right.$ is the radius clearance of bearing) is dimensionless gas-film thickness, $P=p / p_{a}\left(p_{a}\right.$ is the environmental pressure, $\left.p_{a}=10330 \mathrm{~N} / \mathrm{m}^{2}\right)$ is dimensionless gas-film pressure, $\varphi=x / R(x$ is circumferential coordinates, $R$ is the radius of journal ) is dimensionless circumferential coordinates, $\lambda=z / R(z$ is axial coordinates $)$ is dimensionless axial coordinates, $\Lambda=6 \mu \omega R^{2} /\left(p_{a} C_{b}{ }^{2}\right)(\mu$ is dynamic viscosity of gas,$\omega$ is the angular velocity of journal) is bearing number, $T=\omega t$ is dimensionless time, $Q$ is the modified factor that represents rarefaction effect, and $Q$ is the function of gas-film thickness.

To represent the degree of gas rarefaction,the Knudsen number, $K_{n}=\lambda_{0} / h\left(\lambda_{0}\right.$ is the average molecular free path)is introduced.At present,there are three main modified factors, and they can be written as

First-order model[3]:

$Q_{1}=1+6 K_{n}$

Second-order model[5]: 
$Q_{2}=1+6 K_{n}+6 K_{n}^{2}$.

Boltzmann model[9-10]:

$$
Q_{3}=1+0.10842 K_{n}+9.3593 / K n^{-1.17468} \text {. }
$$

For applying MATLAB PDE Toolbox to solve the modified Reynolds equation,the E.q.(1) should be changed into elliptic partial differential equations.

The general form of elliptic partial differential equations can be given as

$$
-\nabla \cdot(c \nabla u)+a u=f
$$

where $\mu$ is variable, $c, a, f$ are constant or variables.

Introducing following notations: $S=P H, \Pi=S^{2}[13]$, the coefficients of elliptic partial differential equations can be obtained

$$
\left\{\begin{array}{l}
c=-1 \\
a=\frac{2}{Q H}\left(\frac{\partial Q}{\partial \lambda} \frac{\partial H}{\partial \lambda}+\frac{\partial Q}{\partial \varphi} \frac{\partial H}{\partial \varphi}+Q \frac{\partial^{2} H}{\partial \lambda^{2}}+Q \frac{\partial^{2} H}{\partial \varphi^{2}}\right) \\
f=\left[\frac{1}{Q}\left(\frac{\partial Q}{\partial \varphi} \frac{\partial \Pi}{\partial \varphi}+\frac{\partial Q}{\partial \lambda} \frac{\partial \Pi}{\partial \lambda}\right)-\frac{1}{H}\left(\frac{\partial H}{\partial \varphi} \frac{\partial \Pi}{\partial \varphi}+\frac{\partial H}{\partial \lambda} \frac{\partial \Pi}{\partial \lambda}\right)\right]
\end{array}\right.
$$

The dimensionless gas-film pressure $\left(\mathrm{P}_{0}\right)$ can be obtained by solving the modified Reynolds equation,therefore the dimensionless load capacity can be given by

$$
C_{L}=\frac{W}{p_{a} R L}=\frac{R}{L} \sum_{i=1}^{3} \int_{-\frac{L}{2 R}}^{\frac{L}{2 R}} \int_{\varphi_{0}}^{\varphi_{1}}\left(P_{0}-1\right) \cos \varphi d \varphi d \lambda .
$$

where $\varphi_{0}$ is the angle of inlet and $\varphi_{1}$ is the angle of outlet. $L$ is the width of bearing. $W$ is the load.

dynamic performance. The pressure distribution in the gas film between the journal and the lobe can be modeled by the following equation, while taking the small disturbance of journal into consideration

$$
\left\{\begin{array}{l}
P=P_{0}+\tilde{P}_{0} e^{i \Omega T} \\
H=\tilde{H}_{0}+\tilde{H}_{0} e^{i \Omega T}
\end{array} .\right.
$$

where $H_{0}$ is static gas-film thickness, $\Omega=v / \omega$ is dimensionless disturbance frequency $(v$ is disturbance frequency of journal).

The amplitude of dynamic gas-film thickness can be represented by following formula:

$$
\tilde{H}_{0}=E_{0} \cos \left(\varphi-\theta_{0}\right)+\varepsilon_{0} \Theta_{0} \sin \left(\varphi-\theta_{0}\right) .
$$

where $E_{0}$ is the amplitude of perturbation eccentricity and $\Theta_{0}$ is the amplitude of perturbation attitude angle.

To apply partial derivative method,introducing following notations: $P_{E}=\frac{\partial \tilde{P}_{0}}{\partial E_{0}} P_{\theta}=\frac{1}{\varepsilon_{0}} \frac{\partial \tilde{P}_{0}}{\partial \Theta_{0}} H_{E}=\frac{\partial \tilde{H}_{0}}{\partial E_{0}} H_{\theta}=\frac{1}{\varepsilon_{0}} \frac{\partial \tilde{H}_{0}}{\partial \Theta_{0}}$.

$P_{\theta}, P_{\mathrm{E}}$ can be obtained by applying MATLAB PDE Toolbox to solve the dynamic equation.

The dynamic performances can be calculated by following formula 


$$
\left\{\begin{array}{l}
-\frac{R}{L} \iint_{A} P_{E} \cos \varphi d \varphi d \lambda=K_{y \varepsilon}+i \Omega_{y \varepsilon} D_{y \varepsilon} \\
\frac{R}{L} \iint_{A} P_{E} \sin \varphi d \varphi d \lambda=K_{x \varepsilon}+i \Omega_{x \varepsilon} D_{x \in}+i \Omega_{y \theta} \\
-\frac{R}{L} \iint_{A} P_{\theta} \cos \varphi d \varphi d \lambda=K_{y \theta}+ \\
\frac{R}{L} \iint_{A} P_{\theta} \sin \varphi d \varphi d \lambda=K_{x \theta}+i \Omega_{x \theta} D_{x \theta}
\end{array} .\right.
$$

Therefore, the dynamic performances of each pad can be calculated by following formula

$$
\begin{aligned}
& {\left[\begin{array}{l}
K_{x x} \\
K_{x y}
\end{array}\right]=\left[\begin{array}{cc}
-\sin \theta_{0} & -\cos \theta_{0} \\
\cos \theta_{0} & -\sin \theta_{0}
\end{array}\right]\left[\begin{array}{l}
K_{x \varepsilon} \\
K_{x \theta}
\end{array}\right]=[A]\left[\begin{array}{l}
K_{x \varepsilon} \\
K_{x \theta}
\end{array}\right],} \\
& {\left[\begin{array}{l}
K_{y x} \\
K_{y y}
\end{array}\right]=[A]\left[\begin{array}{c}
K_{y \varepsilon} \\
K_{y \theta}
\end{array}\right],} \\
& {\left[\begin{array}{l}
D_{x x} \\
D_{x y}
\end{array}\right]=[A]\left[\begin{array}{c}
D_{x \varepsilon} \\
D_{x \theta}
\end{array}\right],} \\
& {\left[\begin{array}{c}
D_{y x} \\
D_{y y}
\end{array}\right]=[A]\left[\begin{array}{c}
D_{y \varepsilon} \\
D_{y \theta}
\end{array}\right],}
\end{aligned}
$$

The whole bearing dynamic stiffness and damping coefficients can be obtained by using numeral superposition method.

\section{Results and discussions}

Applying above method,the algorithms have been used to investigate the effect of rarefaction effect in three-lobe bearing. The initial bearing parameters are presented in Table 1.

Table 1.Bearing parameters.

\begin{tabular}{ll}
\hline Parameters & Three-lobe bearing \\
\hline Bearing radius, $R$ & $0.010[\mathrm{~m}]$ \\
Length-diameter ratio, $L / D$ & 1 \\
Gas dynamic viscosity, $\mu$ & $0.00001833[\mathrm{~Pa} . \mathrm{s}]$ \\
Number of pads, $N$ & 3 \\
Lobe angular extent, $\alpha$ & $\alpha_{1}=\alpha_{2}=\alpha_{3}=110 \pi / 180[\mathrm{rad}]$ \\
Radius clearance, $C_{b}$ & $20[\mu \mathrm{m}]$ \\
\hline
\end{tabular}

static performance.Fig.2shows the dimensionless load capacity versus eccentricity. The results show that the dimensionless load capacity decrease and independent of model,while take the rarefaction into account.But the difference can only be observed when the eccentricity is greater than 0.75.That is because the bigger the eccentricity is, the more obvious the rarefaction effect is. Furthermore, the result of second-order model and Boltzmann model are more consistent,and smaller than the result of first-order model.

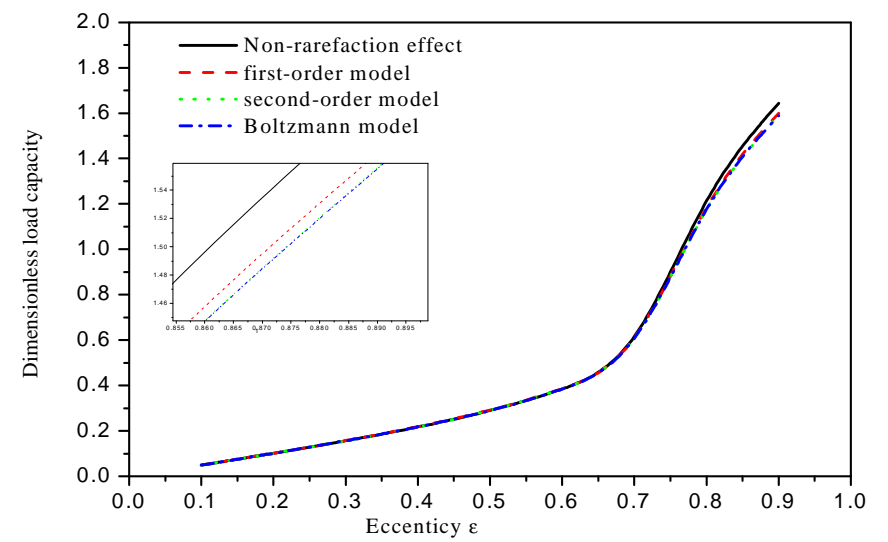

Fig.2 Dimensionless load capacity versus eccentricity $(L / D=1, \Lambda=1)$ 
The relationship of dimensionless load capacity and bearing number is shown in Fig.3As shown in the figure,the dimensionless load capacity is reduced compare with non-rarefaction effect.Moreover,the difference is more obvious when the bearing number is bigger.But the discrepancy in model is negligible.

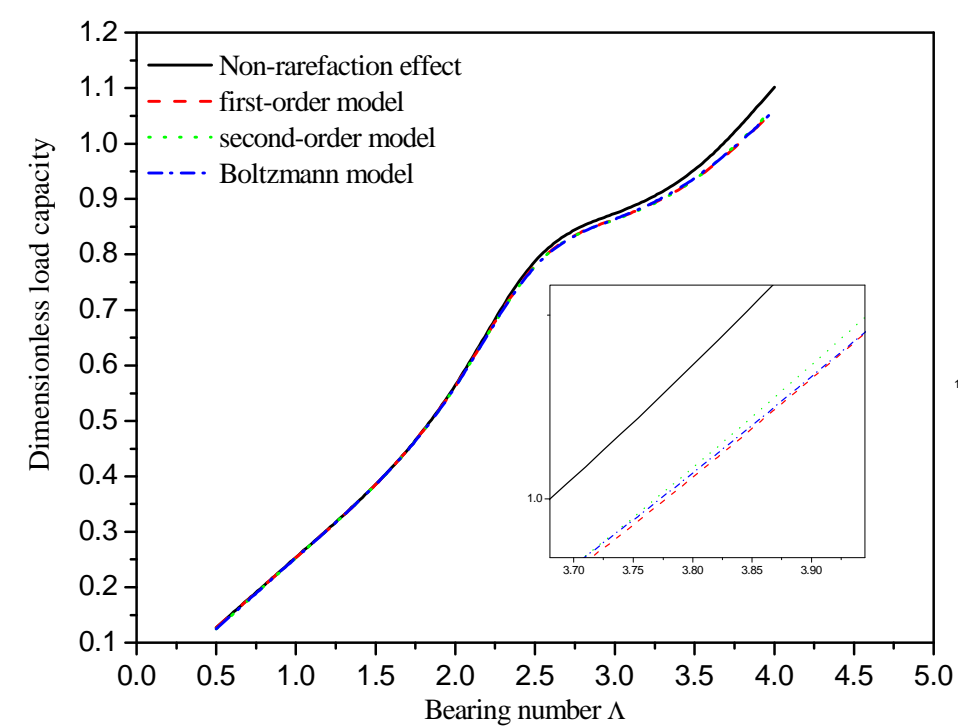

Fig.3Dimensionless load capacity versus Bearing number $(\varepsilon=0.6, L / D=1)$

The dimensionless load capacity versus $L / D$ shown in Fig4.The linear relationship between the dimensionless load capacity and $L / D$ can be seen. When considering the effect of rarefaction,the dimensionless load capacity decrease. Furthermore,the result of second-order model and Boltzmann model are closer.

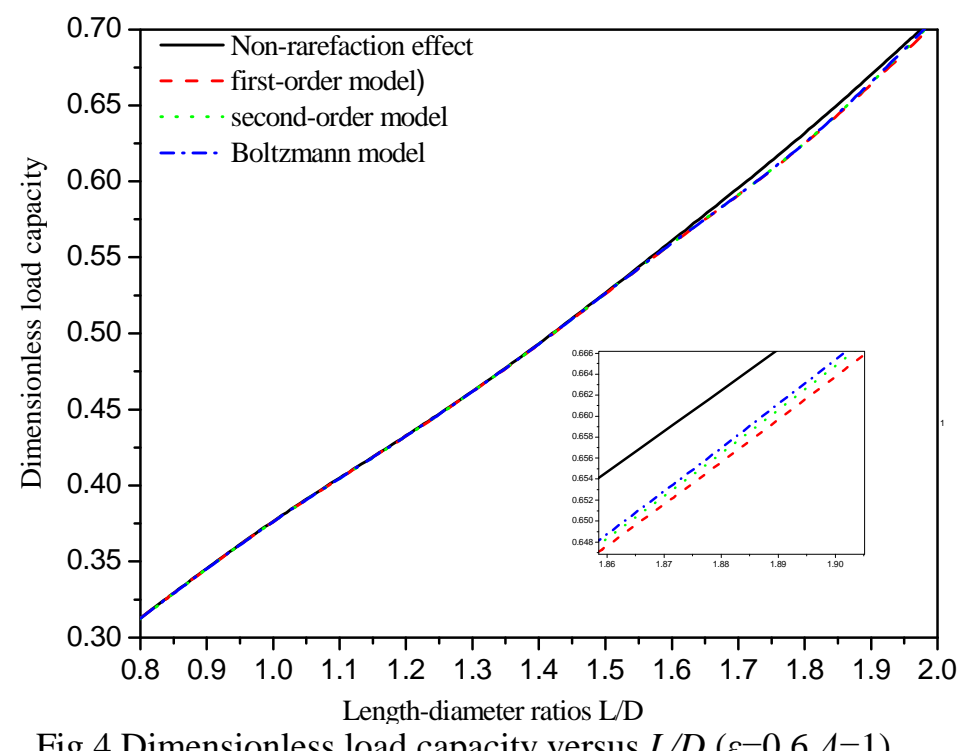

Fig.4 Dimensionless load capacity versus $L / D(\varepsilon=0.6, \Lambda=1)$

dynamic performance.Fig.5 shows fore linear stiffness coefficients versus the eccentricity.As shown in Fig.5,the stiffness coefficients, other than $K_{x y}$,increase along with the increase of eccentricity. When the eccentricity is greater than 0.4 , the stiffness coefficient $K_{x y}$ and $K_{y x}$ become bigger in the case of the rarefaction effect,compare with non-rarefaction effect.But $K_{x x}$ and $K_{y y}$, on the contrary,become smaller.Moreover, the difference of the coefficient $K_{x x}$ increase along with the increase of eccentricity.

Fore linear damping coefficients versus eccentricity is shown in Fig.6.It can be concluded that the coefficients, $D_{y x}$ and $D_{y y}$,increase along with the increase of eccentricity.But $D_{x y}$ and $D_{x x}$ is on the contrary. While taking the rarefaction into account, the coefficients, $D_{y x}$ and $D_{x x}$, are reduced.Compared with the stiffness coefficients, the damping coefficients are insensitive to tha rarefaction effect. 

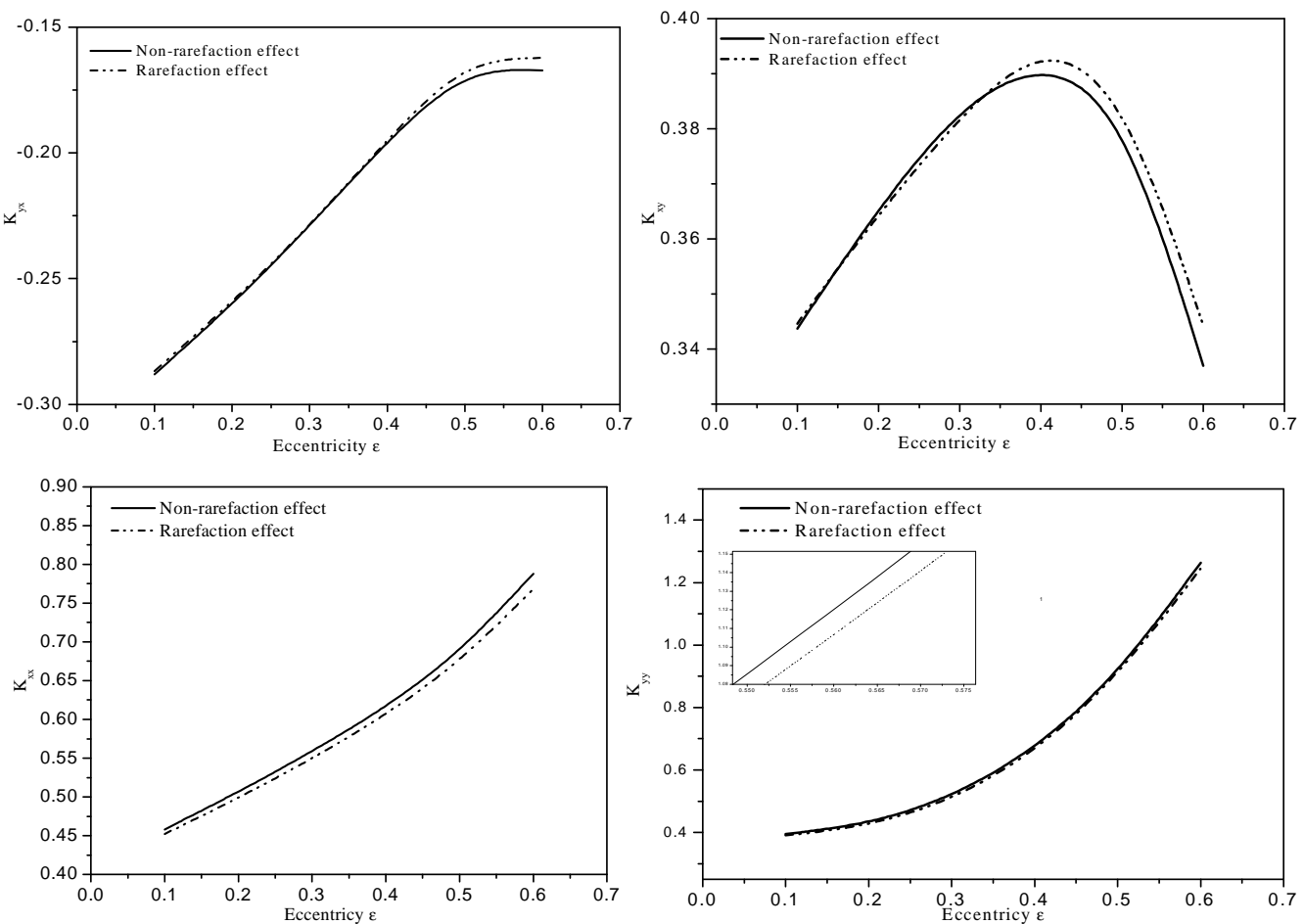

Fig.5 Stiffness coefficients versus Eccentricity $(L / D=1, \Lambda=1, \Omega=1)$
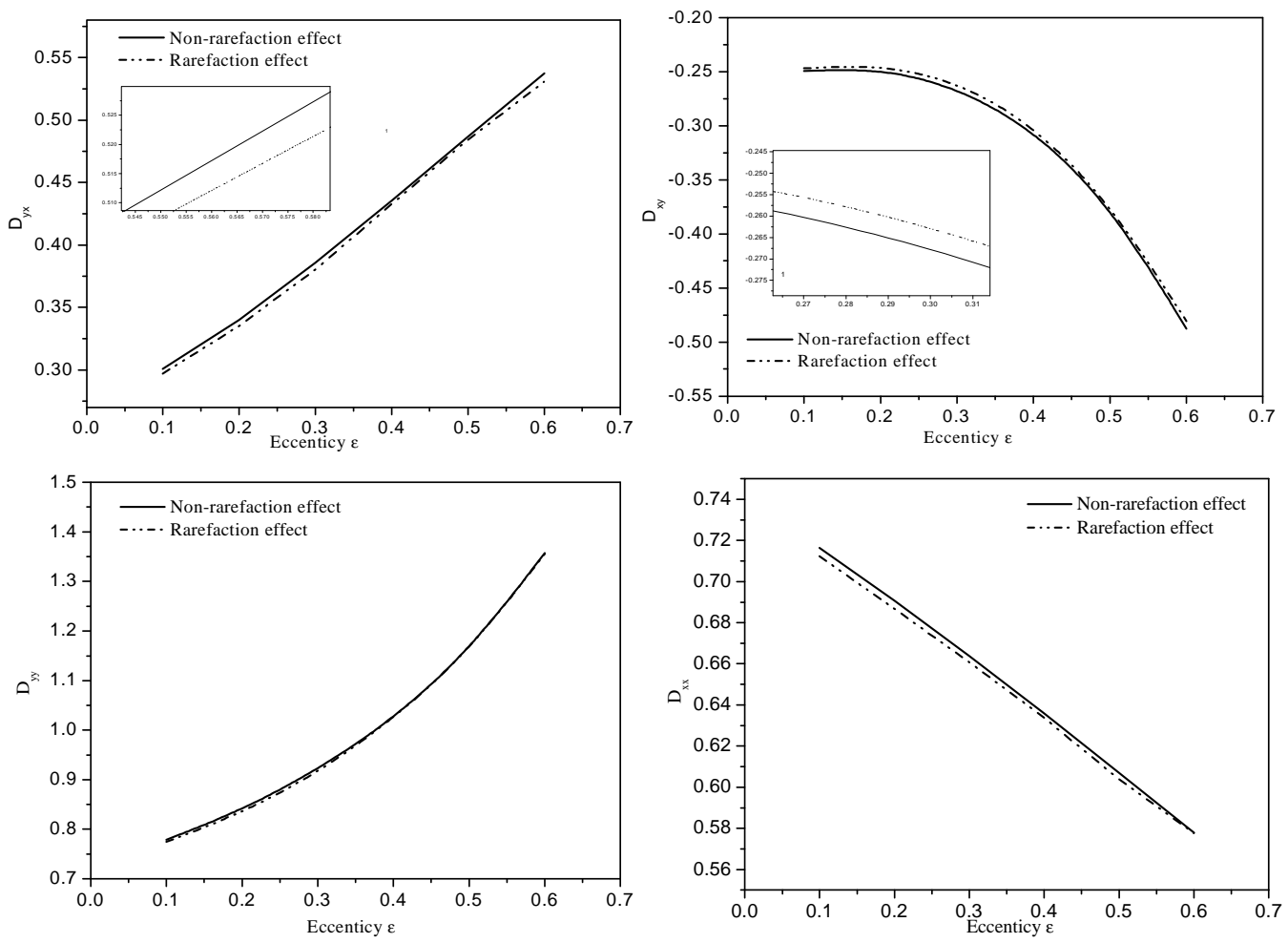

Fig.6 Damping coefficients versus eccentricity $(L / D=1, \Lambda=1, \Omega=1)$

\section{Conclusions}

In this paper,three models,first-order model,second-order model and Boltzmann model,for describing the rarefaction are proposed to modify the Reynolds equation.The static and dynamic performance of the three lobe gas bearing with rarefaction effect are investigated.The result shows:

1.Increasing the value of parameter,such as eccentricity,bearing number, $\mathrm{L} / \mathrm{D}$, the load capacity will be increased .Especially when the eccentricity is bigger than 0.7, the load capacity increase rapidly. 
2.While take the rarefaction effect into consideration, the load capacity decrase. The reduction increase along with the increase of the value of parameter. The results of second-order model are more closer to those of Boltzman model.

3.The direct stiffness coefficients increase along with the increase of eccentricity. While considering the rarefaction effect,the direct stiffness coefficients decrease.But the result of cross stiffness coefficients is inverse,especially when the eccentricity is bigger than 0.4.The damping coefficients are less affected by the rarefaction effect.

\section{Acknowledgements}

This work was financially supported by the National Natural Science Fund (51575425), National Key Basic Research Program (2013CB035706) .

\section{References}

[1] Bhushan B.Tribology and Mechanics of Magnetic Storage Devices[M].New York:Springer,1990.

[2] Gross W A,Matasch L A,Castelli V,et al.Fluid Film Lubrication[M].New York:John Wiley\&Sons, 1980.

[3] Burgdorger A.The influence of the molecular mean free path on the performance of hydrodynamic gas lubrication bearings[J].ASME Journal of Basic Engineering,1959,81;94-100.

[4] LEE Y,KWAK H,KIM C,et al. Numerical predication of slip flow effect on gas-lubricated journal bearing for MEMS/MST-based micro-rotating machinery[J].Tribology International,2005,38:89-96.

[5] Hsia Y T,Domoto G A.An experimental investigation of molecular rarefaction effects in gas lubricated bearings at ultra-low clearances[J].ASME J Tribol,1983,105:120-130.

[6] Mitsuya Y.Modified Reynolds equation for ultra-thin film gas lubrication using 1.5-order slip-flow model and considering surface accommodation coefficient [J].ASME J Tribol,1993,115:289-294.

[7] HUANG Hai,MENG Guang,ZHAO Sanxing. The effects of second-order slip-flow on the steady performance of micro gas bearing[J].Chinese Journal of Theoretical and Applied Mechanics,2006,38 (5):668-673.

[8] ZHANG Wenming,ZHOU Jianbin,MENG Guang.Performance and stability analysis of gas-lubricated journal bearings in MEMS[J]. Tribology International, 2011,44:887-897.

[9] Fukui S,Kaneko R.Analysis of ultra-thin gas film lubrication based on linearized Boltzmann equation:first report-derivation of a generalized lubrication equation including thermal creep flow[J].ASME J Tribol,1988,110:253-262.

[10] Fukui S,Kaneko R.A database for interpolation of Poiseuille flow rates for high Knudsen number lubrication problems[J].ASME J Tribol,1990,112:78-83.

[11] ZHANG Xiaoqing,WANG Xiaoli,LIU Ren,et al.Influence of temperature on nonlinear dynamics characteristics of spiral-grooved gas-lubricated thrust bearing-rotor systems for micro-engine[J].Tribology International,2003,61:138- 143.

[12] ZHANG Xiaoqing,WANG Xiaoli,ZHANG Yuyan.Non-linear dynamic analysis of the ultra-short micro gas journal bearing-rotor systems considering viscous friction effects[J].Nonlinear Dynamics,2013,73:751-765.

[13] Lihua Yang.Study on the performance of aerodynamic tilting-pad and compliant foil bearings[M].Xi'an Jiaotong University,2009. 